\begin{tabular}{|c|c|c|c|}
\hline \multirow{2}{*}{$\begin{array}{c}1901 \\
\text { Sept. } 29\end{array}$} & \multirow{2}{*}{$\begin{array}{l}\text { M. Z. Gr. } \\
8^{\mathrm{h}} 20^{\mathrm{m}}\end{array}$} & $\begin{array}{c}\text { Zahl der } \\
\text { Beob. }\end{array}$ & $\begin{array}{l}\text { Helligkeit } \\
\text { nach H.P. }\end{array}$ \\
\hline & & 4 & 6.75 \\
\hline Oct. I & 72 & 4 & 6.70 \\
\hline I & 91 & 4 & 6.69 \\
\hline 3 & 621 & I & 6.70 \\
\hline 9 & 89 & 5 & 6.59 \\
\hline 10 & 626 & 5 & 6.66 \\
\hline 10 & 954 & 3 & 6.23 \\
\hline 15 & 729 & 3 & 6.68 \\
\hline I 6 & 68 & 3 & 6.70 \\
\hline I 7 & 832 & 4 & 6.60 \\
\hline I 8 & 723 & 3 & 6.42 \\
\hline $2 I$ & 1024 & $\mathbf{I}$ & 6.86 \\
\hline 24 & $\begin{array}{ll}7 & 37\end{array}$ & $\mathbf{I}$ & 6.75 \\
\hline
\end{tabular}

\begin{tabular}{|c|c|c|c|c|}
\hline & M. Z. Gr. & $\begin{array}{c}\text { Zahl der } \\
\text { Beob. }\end{array}$ & $\begin{array}{l}\text { Helligkeit } \\
\text { nach H.P. }\end{array}$ \\
\hline \multicolumn{2}{|c|}{ Oct } & $6^{h} 5^{6^{m}}$ & 2 & 6.69 \\
\hline & 3 I & 559 & 5 & 6.68 \\
\hline Nov & 1 & 547 & 5 & 6.74 \\
\hline & I & 750 & 5 & 6.75 \\
\hline & 2 & 646 & 6 & 6.78 \\
\hline & 3 & 6 I0 & 4 & 6.80 \\
\hline & 3 & 835 & 3 & 6.91 \\
\hline & 4 & 541 & 4 & 6.73 \\
\hline & 4 & 744 & 4 & 6.77 \\
\hline & 5 & 1024 & 3 & 6.65 \\
\hline & 15 & 644 & 3 & 6.78 \\
\hline & I 6 & 6 I I & 5 & 6.76 \\
\hline
\end{tabular}

Bemerkungen.

Der Beobachtungsort war von Aug. 14 bis 26 Borkum, $\varphi=+53^{\circ} 35^{\circ}, \lambda=3^{\mathrm{m}} \cdot 9$ westl. von Münster; sonst immer Münster selbst. Reduction auf Ortszeit $+30^{\mathrm{m}} \cdot 5$.

Als Instrument diente von Aug. 14 bis Sept. 12 ein Steinheil'scher Sucher von $60 \mathrm{~mm}$ Oeffnung, Eigenthum der hiesigen Akademie; sonst immer der früher benutzte Fraunhofer'sche Dreizöller, Eigenthum der kgl. Sternwarte in Berlin.

Vergleichsterne waren:

$\begin{array}{rcccc}\text { Stern } & p & y & u & v \\ \text { Hagen's Nr. } & 1.25 & \text { I. } 38 & \text { II. I } & \text { II. } 5 \\ \text { H. P.-Grösse } & 5.4 & 6.5 & 6.9 & 7: 3\end{array}$

Die Differenz $y-u$ ergiebt sich aus meinen Schätzungen merklich grösser als nach der H.P. Doch sind, da es sich um eine vorläufige Aufstellung handelt, die H. P.-Grössen

Münster, 1901 Nov, 19.

zur Rechnung einfach beibehalten. Der Veränderliche wurde immer an $y$ und $u$ angeschlossen, in helleren Phasen auch noch an $\not$, in schwächeren an $v$. Im Text ist die $Z_{a g h l}$ der vollständigen Beobachtungen mitgetheilt, so dass z. B. Nov. 2 zur Mittelbildung $6 \times 3$ Einzelschätzungen benutzt sind, indem der Stern jedesmal an $y, u$ und $v$ angeschlossen war.

Im August und September war die Abnahme der Hellig: keit im Grossen bemerkbarer als die kleine Oscillation. Oct. 10 ist gerade bei der zweiten, ein deutliches Aufflammen ergebenden Gruppe besonders gute Luft notirt. Weitere Maxima fanden statt Oct. I 8, Luft ziemlich gut; Oct. 30-3 I, Luft am 30. schlecht, am 31 . fast immer gut; Nov. 5, sehr deutliches Aufflammen bei guter Luft.

Für genauere Berechnung steht eine Abschrift des Originals zur Verfügung.

Ұ. Plassmann.

\title{
Altra „Notiz betr. die Eigenbewegung der Nova Persei (Ch. 1226)“.
}

In relazione alle osservazioni di L. Courvoisier in Heidelberg (vedi A.N. 3753), credo opportuno comunicarle senza indugio il confronto istituito anche da me fra le osservazioni fatte al Piccolo Meridiano in Febbraio (vedi A.N. 3698) e quelle nell' Ottobre, per la Nova Persei:

\begin{tabular}{|c|c|c|}
\hline 1901 & $\alpha 1901.0$ & 81901.0 \\
\hline $\begin{array}{lrl}\text { Febbraio } & 25,26,27 \\
\text { Ottobre } & 8,10,11\end{array}$ & $\begin{array}{lll}3^{\mathrm{h}} & 24^{\mathrm{m}} & 28.08 \\
3 & 24 & 28.12\end{array}$ & $\begin{array}{l}+43^{\circ} 33^{\prime} 54^{\prime \prime} \circ \\
+433353.8\end{array}$ \\
\hline
\end{tabular}

Arcetri-Firenze, 1901 Dic. 17.

B. Viaro.

Planet (118) Peitho. Corr. der Ephemeride (Berl. Jahrb. 1904): Dec. $3^{1}+1^{m} 4^{3}-1: 0 . \quad W$. Luther.

Planet (230) Athamantis. Dec. $287^{\mathrm{h}} 6^{\mathrm{m}} \cdot 3 \mathrm{M}$. Z. Heidelbetg $4^{\mathrm{h}} 48^{\mathrm{m}} \cdot{ }^{\mathrm{m}}+16^{\circ} 13^{\prime}$. Kopff.

Planet 1901 HL. Dec. $287^{\mathrm{h}} 6^{\mathrm{m}} \cdot 3$ M. Z. Heidelberg $4^{\mathrm{h}} 4 \mathrm{r}^{\mathrm{m}} \cdot 8+16^{\circ} 3^{2^{\mathrm{t}}}$. Kopff.

Anforderang betr. Beobachtungen des Cometen 1901 I. Herr C. F. Merfield, Royal Society, Elizabeth Street 5 , Sydney, beabsichtigt die Bahn des Cometen rgor I zu berechnen und bittet um Mittheilung von noch unpublicirten Beobachtungen.

Personalnotiz. Dr. R. Schorr ist zum Professor und Director der. Sternwarte in Hamburg ernannt worden.

\footnotetext{
Inhalt zu Nr. 3760. E. E. Barnard. On the Dimensions of the Planets and Satellites. 26I. - F. Schwab. Beobachtungen des Cometen I9oo II. 267. - H. Struve. Beobachtungen des Cometen Igoo II. 267. - E. Hartwig. Beobachtungen des Encke'schen Cometen 1901 II. 269. - K. Graff. Berichtigungen zu Veröff. R. I. Nr. 14. 269. - W. Luther. Beobachtung des Planeten Igor HJ. 269. - E. Millosevich. Elementi ed effemeride di (303) Josephina. 271. - G. Boccardi. Elementi ed effemeride del pianeta (347) Pariana. 271. - F. Plassmann. Ueber die Nova Persei (Ch. 1226). 273. - B. Viaro. Altra $\rightarrow$ Notiz betr. die Eigenbewegung der Nova Persei (Ch. 1226)火. 275. - Kleine Mittheilungen. 275. 